\title{
RESUMEN.
}

En las últimas décadas, en distintos países se han producido reformas significativas en el sector público, siendo la principal innovación la convergencia de la contabilidad gubernamental hacia las Normas Internacionales de Contabilidad para el Sector Público (NICSP). Estas normas reflejan la esencia económica de las operaciones del negocio, presentando una imagen fiel de la situación financiera de una entidad de una manera estándar. Esta investigación se llevó a cabo con una metodología cualitativa, con un alcance comprehensivo respecto a casos, con el fin de conocer la contabilización en base a NICSP de los seres vivos correspondientes a los animales del zoológico que están en exhibición, agregando el efecto que tuvo la identificación de los bienes de uso de la entidad, conociendo las debilidades, fortalezas, tareas y procedimientos que se presentaron en este proceso. Para cumplir con los objetivos expuestos se realizarán entrevistas al área de Contabilidad del Servicio Público, al igual que a expertos en el tema con estudios en NICSP, y en Biología. Finalmente, se ha visto que el organismo en estudio ha llevado las etapas del plan de convergencia mediante la capacitación al personal y consultas a la Contraloría General de la República.

Palabras Claves: normas internacionales de contabilidad del sector público, activos, bienes de uso, medición, inventario, vida útil.

\section{"ANÁLISIS DE LA IMPLEMENTACIÓN DE LAS NORMAS INTERNACIONALES DE CONTABILIDAD PARA EL SECTOR PÚBLICO: ENFOCADO AL ESTUDIO DE BIENES DE USO DE UNA ENTIDAD PILOTO"}

Marcela Fernández Rozas 1 / Joselyn Dennise Aguayo Mondaca²

\section{"ANALYSIS OF THE IMPLEMENTATION OF THE INTERNATIONAL STANDARDS OF ACCOUNTING FOR THE PUBLIC SECTOR: FOCUSED ON THE FIXED ASSETS OF USE OF A PILOT ENTITY"}

\section{ABSTRACT}

In recent decades, significant reforms have taken place in the public sector in different countries, being the main innovation the recent convergence of government accounting towards International Accounting Standards for the Public Sector. These standards reflect the essence of business operations, presenting a true picture of the financial situation of an entity in a standard way. This research was carried out with a qualitative methodology, with a comprehensive scope with respect to cases, in order to know the accounting based on Accounting Standards for the Public Sector of the living beings corresponding to the animals of the zoo that are on display. Adding the effect that the identification of the fixed assets of the entity had, knowing the weaknesses, strengths, tasks and procedures that were presented in this process. In order to comply with the stated objectives, they will conduct interviews to the area of Public Service Accounting, experts in the field with studies in IPSAS, and in Biology. Finally, concluding that the organism under study has carried out the stages of the plan of convergence through staff training and consultations with the Office of the Comptroller General of the Republic.

Key Words: International accounting standards of the public sector, Assets, fixed assets, measurement, inventory, useful life.

${ }^{1}$ Contador Auditor, Magister en Gestión de Organizaciones, Mención Finanzas, Escuela de Auditoría, Universidad de Valparaíso. marcela.fernandez@uv.cl

${ }^{2}$ Licenciada en Sistemas de Información Financiera y Control de Gestión. joselyn.aguayo@alumnos.uv.cl 
Revista de Investigación Aplicada en Ciencias Empresariales 


\section{INTRODUCCIÓN}

Tomando en cuenta la adaptación de las normas internacionales de Contabilidad del Sector Público (NICSP), esta investigación se enfocará en el estudio de una de las entidades piloto: El Parque Nacional Metropolitano, la cual tuvo que adecuar y presentar sus estados financieros bajo esta modalidad. Desde esto entonces, nace la interrogante y necesidad de conocer qué normativa actual aplica esta organización para contabilizar a los seres vivos. Estos animales pueden ser considerados activos biológicos bajo la NICSP 27 de agricultura, sin embargo, no son producidos para la venta, por lo que se presume de corroboración para este caso tan inusual, donde los animales son utilizados solo como exhibiciones. Entonces, se necesita saber qué normativa se aplica, para saber si pueden ser considerados activos fijos.

Como objetivo general se pretende analizar contablemente, bajo la normativa, el reconocimiento de seres vivos en un servicio público piloto (Parque Metropolitano) de la región de Santiago, en los periodos 2016-2017. Posteriormente, los objetivos específicos en la investigación serán planteados sobre aspectos más concretos, derivados del objetivo general. El primero sería identificar las normativas asociadas a los bienes considerados como activos materiales en la Norma Internacional de Contabilidad para Sector Público; segundo, se deben reconocer las particularidades de los bienes materiales identificados como "seres vivos", bajo la normativa internacional; y por último, se tiene que describir el proceso de medición inicial y posterior de los animales en exhibición efectuado por el servicio público en estudio.

\section{METODOLOGÍA}

El presente estudio es de Enfoque Cualitativo, con un alcance comprehensivo con respecto a casos. En esta investigación se realizó una entrevista semi estructurada en la cual, a través de una pauta inicial, se desarrolló un diálogo, donde se permitió integrar otras preguntas de acuerdo al itinerario que seguía la conversación. Este diálogo se grabó en un archivo de audio, con el previo consentimiento del entrevistado.

Para el caso de la entrevista a informantes claves, se seleccionó a un organismo por estrato considerando los siguientes criterios: que sea un Servicio Público y una entidad piloto, que se encuentre ubicado físicamente en Santiago, que esté en el proceso de implementación de la Resolución Nro.16 (emitida por la Contraloría General de la República), y que se pueda obtener acceso a la información requerida para llevar a cabo la investigación. También se seleccionó una persona de un organismo público con las siguientes características: que trabaje en las áreas de Contabilidad y Finanzas de la entidad en estudio, que dentro de sus funciones realice actividades directamente relacionadas con bienes de uso, que tenga experiencia y conocimiento en el tema, y que tenga capacitaciones de NICSP. 
De acuerdo al problema de investigación, la información será distribuida según el marco teórico, a través de narrativas y el uso de cuadros comparativos y figuras; iniciando con aspectos generales del tema en estudio, para terminar con aspectos más específicos en los cuales se enfocará la problemática. La propuesta metodológica para la entrevista incorpora los criterios de credibilidad, confirmabilidad, fiabilidad y transferencia.

Respecto al análisis de los resultados que se obtuvieron en las entrevistas, estos se transcribieron de acuerdo a categorías de análisis. Para el análisis de los resultados, respecto a la Normativa Internacional de la Contabilidad para el sector público, fue necesario realizar la transcripción de la información recopilada por medio de este instrumento, para poder facilitar el análisis e interpretación.

Finalmente, para la discusión de resultados se realizó una comparación de estos mismos, con la teoría que sustenta a la investigación. Las conclusiones finales se realizaron en función de los objetivos específicos propuestos inicialmente.

\section{MARCO TEÓRICO}

\section{NICSP A NIVEL INTERNACIONAL}

\section{Antecedentes Generales}

El Consejo de Normas Internacionales de Contabilidad del Sector Público (IPSASB) desarrolla las Normas Internacionales de Contabilidad para el Sector Publico (NICSP) las cuales establecen los requerimientos para la información financiera de los gobiernos y otras entidades del sector público. Las NICSP presentan las mejores prácticas internacionales de información financiera para su aplicación a entidades públicas de distintos países, específicamente en los estados financieros.

La Federación Internacional de Contadores (IFAC) es la organización a nivel mundial para la profesión contable, que cumple con la misión de servir al interés público, fortalecer la profesión contable en todo el mundo y contribuir al desarrollo de economías internacionales fuertes. Esto se logra a través de diversas acciones como el establecer y promover la observancia de normas profesionales de calidad profesionales de calidad, el favorecer la convergencia internacional de tales normas y el expresar temas de interés púbico donde la experiencia de la profesión es más relevante.

Chile en este escenario ha decidido efectuar una adopción indirecta de las NICSP, emitiendo una norma nacional que cumpla con los requerimientos de estas, Io que permite alcanzar e incrementar calidad, comparabilidad y transparencia de la información financiera y contable, además de obtener una mejora en la rendición de cuentas de las entidades públicas. La Contraloría de la Nación ha estado trabajando en mejoras y actualizaciones a la normativa que rige al sector público. Al mismo tiempo, esta actúa como entidad fiscalizadora destinada a ejercer el control de 
legalidad de los actos administrativos, que serían los ingresos e inversiones de fondos fiscales, municipales y otros. A su vez, se encarga de asegurar el cumplimiento de los principios y normas contables, y los procedimientos por el cual, de forma obligatoria, se rige el sistema de contabilidad general de la Nación.

En el año 2012, se seleccionaron doce servicios públicos a ser parte del plan piloto del Sistema de Contabilidad Gubernamental con las NICSP, que representarían al sector público con el objetivo de monitorear y orientar la adaptación al nuevo sistema normativo contable. Finalmente, enfrentarían el gran desafío en el año 2016, el cual era presentar sus primeros estados financieros bajo las NICSP de acuerdo al Oficio 54977 del 2010. El desafío tiene como propósito definir y homogeneizar la estructura de estos informes, los cuales entregan información que determinan la situación financiera, presupuestaria y patrimonial de cada organismo.

Los organismos que tuviesen mayor dificultad para proceso de implementación, respecto a la presentación de los Estados Financieros, pudieron optar con más plazo hasta el 2018. Actualmente, este proceso se enfoca en la implementación de la guía práctica de regularización de activos de uso de la organización, y adicionalmente se llevan a cabo seminarios de seguimiento con el objetivo de garantizar una verificación adecuada y eficiente de los bienes patrimoniales. A lo largo de este año, fue necesario realizar un conjunto de acciones de regularización de la información contable actual de las entidades y de fortalecimiento de capacitaciones; igualmente se espera que se siga aplicando este funcionamiento el próximo año.

\section{Federación Internacional de Contadores (IFAC)}

La Federación Internacional de Contadores fue fundada en 1977, definida como la organización mundial para la profesión del contador, con la misión de "servir al interés público, fortaleciendo de forma continuada la profesión contable en todo el mundo y contribuyendo al desarrollo de economías internacionales fuertes estableciendo y promoviendo la observancia de normas profesionales de alta calidad, impulsando la convergencia internacional de estas normas y tomando postura en el interés público allá donde la experiencia de la profesión sea más relevante". (Federacion Internacional de Contadores , 2008, p.1)

\section{Consejo de Normas Internacionales de Contabilidad del Sector Público (IPSASB)}

El IPSASB es un consejo responsable del desarrollo de normas contables y directrices bajo su propia autoridad, siempre que estén dentro de los términos establecidos, para que utilicen las entidades del Sector Público en la preparación de estados financieros. Estos Informes con propósito general deben ser emitidos para usuarios que no solicitan información financiera para satisfacer sus necesidades específicas de antecedentes para la toma de decisiones. 


\section{Normas Internacionales de Contabilidad del Sector Público (NICSP)}

La Federación Internacional de Contadores define: "Las NICSP son normas generales de información financiera de alta calidad para su aplicación a entidades del sector público excepto las empresas públicas". (Federacion Internacional de Contadores, 2008, p. 2).

\section{NICSP Vigentes}

Las NICSP emitidas por el IPSASB mayoritariamente se han basado en los Internacional Financial Reporting Standards (IFRS), atendiendo a algunas de las especificidades propias del sector público. Tal circunstancia se sintoniza con el objetivo de convergencia internacional hacia un único conjunto de estándares de alta calidad, que es una de las premisas operativas del IPSASB. Al 2016, han sido emitidas treinta y dos (32) normas para el proceso de armonización, de las cuales seis no tienen como base ninguna IFRS, por referirse a transacciones 0 particularidades del Sector Publico.

\section{Premisas Básicas}

Las premisas básicas que las NICSP se basan son dos, Base Devengado y Ente en Marcha:

$\checkmark$ Base Devengada: Los efectos de las transacciones y demás sucesos de incidencia económica financiera se reconocen cuando ocurren y afectan cualitativa 0 cuantitativamente el patrimonio de las entidades, independientemente de cuándo se recibe 0 paga el dinero u otro equivalente a efectivo. Lo anterior quiere decir que los EEFF elaborados y presentados sobre la base del devengado informan a los usuarios no solo transacciones pasadas que suponen ingresos 0 egresos, si no también las obligaciones de pago y los derechos de cobro futuro.

$\checkmark \quad$ Ente en Marcha: Los EEFF se elaboran sobre la base en la que el ente contable está en funcionamiento y que continuará sus actividades de operación financiera dentro de un futuro previsible, por esto, se asume que la entidad de gobierno no tiene intención de liquidar o recortar de forma importante sus actividades.

\section{Relación entre las NICSP y las Normas Internacionales de Información Financiera (NIIF)}

Actualmente las NICSP se desarrollan con base contable de acumulación 0 devengado, en el cual convergen con las Normas Internacionales de Información Financiera (NIIF) del sector privado, siendo las primeras adaptadas por el IPSASB de manera tal que buscan mantener el tratamiento contable y texto original de las NIIF, emitidas estas por el IASB, siempre y cuando no existan razones significativas y necesarias que justifiquen 
una aplicación de estas normas para reflejar de mejor forma la situación de alguna entidad del sector público, estas son llamadas "empresas públicas".

La NICSP define empresa pública como "la entidad que reúne todas las siguientes características: es una entidad que tiene la facultad de contratar en su propio nombre, se le ha asignado capacidad financiera y operativa para llevar a cabo una actividad, en el normal curso de su actividad vende vienes y presta servicios a otras entidades obteniendo un beneficio 0 recuperando el costo total de los mismos, no depende de una financiación continua".

Por otra parte, las NICSP desarrollan más exhaustivamente materias inherentes al sector público sobre aspectos de información financiera que no son tratados a profundidad por las NIIF existentes, o para las materias que el IASB no han desarrollado normativa alguna.

También IPSASB ha emitido una NICSP global cuya base contable es de efectivo, esta se encuentra en el Manual de Pronunciamientos del IPSASB e incluye secciones de carácter obligatorias y otras recomendadas sobre información a revelar.

El siguiente cuadro Nro. 1, presenta una comparación entre el ámbito del Sector Público y el Sector Privado, indicando además los organismos emisores internacionales de las normas y los organismos encargados de regularlas en Chile.

\section{Cuadro N¹: Paralelo entre los Procesos del Sector Público y del Sector Privado}

\section{PARALELO ENTRE LOS PROCESOS DEL SECTOR PÚBLICO Y DEL SECTOR PRIVADO:}

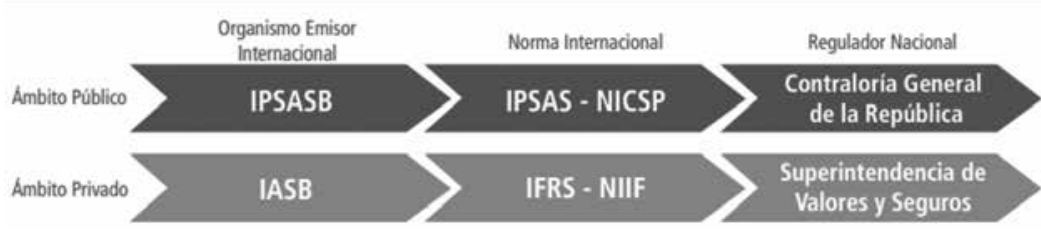

Fuente: Boletín Nro. 1 de la C.G.R. (2012)

\section{NORMATIVA DE INFORMACIÓN CONTABLE DEL SECTOR PÚBLICO EN CHILE}

\section{Plan de convergencia}

En el 2010, la CGR decide iniciar un proceso regulado de convergencia a NICSP, que tiene como objetivo definir la cobertura del proceso, la metodología de la convergencia normativa, las acciones de difusión, los mecanismos de capacitación y la adecuación de los sistemas informáticos. Los desafíos propuestos eran, tal como se muestran en la figura $\mathrm{N}^{0} 1$, los siguientes: 


\section{Figura N¹: Desafíos Propuestos por el Plan de convergencia}

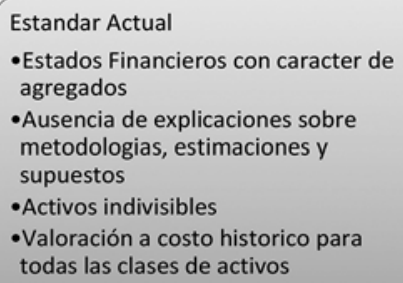

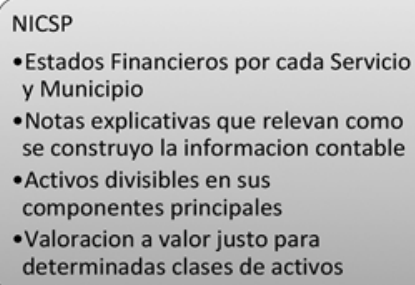

Fuente: Elaboración Propia (2017), respecto a información de la Contraloría General de la República.

Se emitió un calendario de implementación, desde el 2010 hasta 2015 que explica el proceso de la realización de acciones que adaptaron todas estas normas internacionales emitidas. En el 2016 se inicia la aplicación oficial de toda normativa completa para los servicios públicos, que presentarían sus primeros estados financieros bajo esta normativa.

\section{- Entidades piloto}

En la primera etapa del proceso de implementación se conformó un grupo de organizaciones públicas, denominadas "entidades piloto", donde su función principal fue ser contraparte del análisis y evaluación del impacto que trajo este cambio normativo en el sector público, comenzado año 2015.

Las entidades pilotos seleccionadas para este desafío están nombradas en la figura Nro. 2.

\section{Figura N²: Lista de Entidades Piloto}

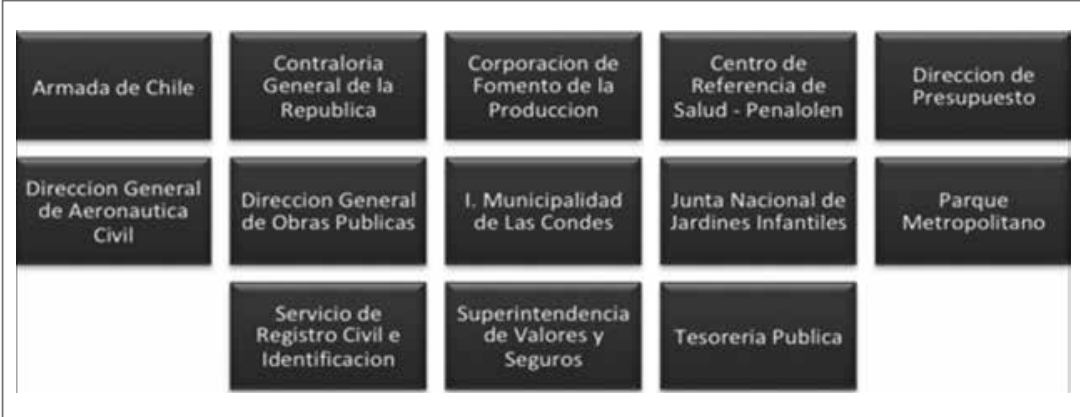

Fuente: Elaboración Propia (2017), a partir del Boletín NICSP Nro.1 (2012) 


\section{- Contraloría General de la República (CGR)}

La contraloría General de la República como entidad fiscalizadora superior de Chile, que tiene un carácter autónomo y rango constitucional, es la encargada de ejercer control de legalidad de los actos de la Administración Pública, y otras funciones más a su cargo, y en particular llevar la contabilidad general de la Nación, de acuerdo lo que se establece en la Constitución Política del Estado y lo regulado en la Ley Orgánica Nro. 10.336 .

La CGR tiene una labor indispensable para el funcionamiento ya que, como ente fiscalizador de carácter jurídico, contable, y financiero, está destinada a velar por el principio de legalidad, es decir, verifica que los órganos de la Administración del Estado actúen dentro del ámbito de sus atribuciones y con sujeción a los procedimientos que la ley contempla.

Su misión se basa en velar por el cumplimiento del ordenamiento jurídico por parte de la Administración del Estado, a través de una relación colaboradora con sus organismos, promoviendo el bien común mediante una gestión institucional eficiente en resguardo de la probidad, la transparencia y el correcto uso del patrimonio público.

\section{- Sistema de Contabilidad General de la Nación}

El Sistema de Contabilidad General de la Nación está constituido según la CGR año 2005 por "un conjunto de principios, normas, y procedimientos técnicos, que permiten el registro de los hechos económicos inherentes a las operaciones del Estado, con el objeto de satisfacer las necesidades de información para apoyar el control y el proceso de toma de decisiones de los administradores y para el conocimiento de terceros interesados en la gestión." (Federación Internacional de Contadores, 2008, p. 3).

\section{NORMATIVA EN PARTICULAR}

1. NICSP 17: Propiedad, Planta y Equipo

\section{- Alcance}

Se aplica a:

$\checkmark$ Todas las entidades públicas que no sean empresas públicas.

$\checkmark$ A los elementos componentes de la propiedad, planta y equipo incluyendo: equipamiento militar especializado, y activos de infraestructura.

$\checkmark$ A los elementos individuales de la propiedad, planta, y equipo, usados para desarrollar o mantener las actividades en bosques. 
No se aplica a:

$\checkmark$ Bosques y recursos naturales renovables similares.

$\checkmark$ Derechos mineros, la explotación para la extracción de minerales, petróleo, gas natural u otros recursos no renovables similares.

$\checkmark \quad$ Cuando otras NICSP u otra guía internacional de relevancia permita determinar la valorización inicial en libros de los elementos de la propiedad, planta y equipo con un enfoque diferente.

$\checkmark \quad$ Cuando no aborda ciertos aspectos de la aplicación para reflejar los cambios en los precios cuando hay una economía Hiperinflacionaria.

\section{- Bienes del Patrimonio Histórico artístico y/o cultural}

Esta norma no requiere que una entidad reconozca los bienes del patrimonio histórico artístico y/o cultural, debido a que su importancia cultural, medioambiental o histórica presentan determinadas características:

$\checkmark$ Es poco probable que su valor en términos culturales, medioambientales, educacionales e históricos quede perfectamente reflejado en un valor financiero basado en el mercado.

$\checkmark$ Las obligaciones legales $\mathrm{y} / 0$ estatutarias pueden imponer prohibiciones o severas restricciones a su disposición por venta.

$\checkmark$ Son a menudo irremplazables y su valor puede incrementarse con el tiempo, incluso sí sus condiciones físicas se deterioran.

$\checkmark \quad$ La vida útil puede ser difícil de estimar, en la cual hay casos que pueden ser cientos de años.

Algunos bienes del patrimonio histórico y/o cultural tienen un potencial de servicios, por ejemplo, un edificio histórico artístico puede ser utilizado para oficinas, en estos casos pueden ser reconocidos o medidos.

La información a revelar requiere que las entidades den información sobre los activos contabilizados, por lo tanto, las entidades que reconocen bienes del patrimonio histórico artístico y/o cultural deben exponer en relación con dichos puntos tales como:

$\checkmark \quad$ La base de medición utilizada.

$\checkmark \quad$ El método de amortización utilizado, si los hubiere.

$\checkmark \quad$ El valor contable bruto.

$\checkmark \quad$ La amortización acumulada al final del período si la hubiere. 
$\checkmark$ Conciliación entre el valor en libros al comienzo y al final del período mostrando determinados componentes de la misma.

\section{- Reconocimiento}

Un elemento de la propiedad, planta y equipo deberá reconocerse como un activo cuando:

$\checkmark \quad$ Es probable que la entidad obtenga futuros beneficios económicos o servicio potencial derivados del activo.

$\checkmark \quad$ El costo del activo para la entidad pueda ser medido con suficiente fiabilidad.

La propiedad, planta y equipo son en general la mayor parte de los activos de una entidad y, por lo tanto, resultan significativos en el contexto de su situación financiera. El reconocimiento como es de forma inmediata, puesto que la transacción que pone de manifiesto la compra del activo identifica su costo.

En el caso de un activo construido por la propia entidad, una medida fiable del costo puede venir dada por las transacciones con terceros ajenos a la entidad, para adquirir los:

$\checkmark$ Materiales,

$\checkmark \quad$ Mano de Obra, y

$\checkmark \quad$ Otros factores consumidos durante el proceso de construcción.

Además, bajo determinadas circunstancias el costo se determina por referencia a su valor razonable.

\section{- Medición Inicial de los Elementos de la Propiedad, Planta y Equipo}

Todo elemento de la propiedad, planta y equipo, que cumpla las condiciones para ser reconocido como un activo, debe ser medido inicialmente por su costo.

Una entidad puede recibir una propiedad, planta y equipo como regalo 0 donación por un costo nulo o insignificante; bajo esas circunstancias el costo es su valor razonable en la fecha de su adquisición y no constituye una revaluación.

\section{- Componentes del Costo}

El costo de los elementos de la propiedad, planta y equipo comprende su precio de compra, incluidos: 
$\checkmark \quad$ Los aranceles de importación.

$\checkmark$ Los impuestos indirectos no recuperables que recaigan sobre la adquisición.

$\checkmark$ Cualquier costo directamente relacionado con la puesta en servicio del activo para el uso al que está destinado.

Se deducirá cualquier eventual descuento o rebaja del precio para llegar al costo del elemento.

Cuando se difiere el pago de un elemento integrado a la propiedad, planta y equipo, más allá de los plazos normales del crédito comercial, su costo será el precio equivalente al contado. La diferencia entre esta cantidad y los pagos totales se reconocerá como costos por intereses a lo largo del periodo del aplazamiento.

Los costos de administración, así como otros gastos indirectos de tipo general, no constituyen un componente del costo de la propiedad, planta y equipo, salvo que estuviesen relacionados directamente con la adquisición del activo.

El costo de un activo construido por la propia entidad se determina utilizando los mismos principios que para los activos adquiridos del exterior.

\section{- Intercambio de Activos}

El elemento puede ser adquirido por medio de un intercambio total 0 parcial con otro elemento distinto del activo fijo, u otro activo cualquiera. El costo de adquisición del elemento se medirá por el valor razonable del activo recibido, que equivale al valor razonable del elemento entregado.

Asimismo, puede ser adquirido un elemento mediante un intercambio por otro activo similar, con un uso parecido dentro de la misma línea de actividad y con un valor similar al entregado. También puede ser vendido a cambio de obtener derechos de propiedad sobre activos similares. En ambos casos no se reconocen pérdidas ni ganancias en la transacción.

\section{- Revaluaciones}

El valor razonable de los terrenos y edificios será su valor de mercado; este valor se determinará por medio de una tasación, el valor razonable será fácilmente determinable por referencia a precios establecidos en un mercado líquido y activo. En caso contrario puede establecerse el valor razonable en referencia a otros elementos de características similares, en circunstancias y ubicación similares. 


\section{- Depreciación}

Se depreciará de forma separada cada parte de un elemento de Propiedades, Planta y Equipo que tenga un costo significativo con relación al costo total del elemento. El cargo por depreciación de cada periodo se reconocerá en el resultado (ahorro 0 desahorro), salvo que se haya incluido en el importe en libros de otro activo. Sin embargo, en ocasiones los beneficios económicos o potenciales de servicio futuros incorporados a un activo son absorbidos en la producción de otros activos. En este caso, el cargo por depreciación formará parte del costo del otro activo y se incluirá en su importe en libros.

El importe depreciable de un activo se distribuirá de forma sistemática a lo largo de su vida útil. El valor residual y la vida útil de un activo se revisarán, como mínimo, al término de cada periodo anual sobre el que se informe y, si las expectativas difirieren de las estimaciones previas, los cambios se contabilizarán como un cambio en una estimación contable, de acuerdo con la NICSP 3 Políticas Contables, Cambios en las Estimaciones Contables y Errores.

La depreciación se contabilizará incluso si el valor razonable del activo excede a su importe en libros, siempre y cuando el valor residual del activo no supere al importe en libros de este. Las operaciones de reparación y mantenimiento de un activo no evitan realizar la depreciación, por el contrario, algunos activos pueden tener un mantenimiento inadecuado 0 demorado indefinidamente debido a limitaciones presupuestarias. Cuando las políticas de gestión de los activos empeoren el desgaste y uso de un activo, su vida útil debería estimarse nuevamente y reajustarse según el caso.

El importe depreciable de un activo se determina después de deducir su valor residual. En la práctica, el valor residual de un activo a menudo es insignificante y, por tanto, irrelevante en el cálculo del importe depreciable. El valor residual de un activo podría aumentar hasta igualar o superar el importe en libros del activo. Si esto sucediese, el cargo por depreciación del activo sería nulo, a menos que ese valor residual disminuyera posteriormente y se hiciera menor que el importe en libros del activo.

La depreciación de un activo comenzará cuando esté disponible para su uso, esto es, cuando se encuentre en la ubicación y en las condiciones necesarias para ser capaz de operar de la forma prevista por la gerencia. La depreciación de un activo cesará cuando el activo sea dado de baja. En consecuencia, la depreciación no cesará cuando el activo esté sin utilizar o se haya retirado del uso activo, a menos que se encuentre depreciado por completo. Sin embargo, si se utilizan métodos de depreciación en función del uso, el cargo por depreciación podría ser nulo cuando no 
tenga lugar ninguna actividad de producción.

La vida útil de un activo se definirá en términos de la utilidad que se espere que aporte a la entidad. La política de gestión de activos de una entidad puede implicar la disposición de activos después de un tiempo específico de uso, o tras haber consumido una cierta proporción de beneficios económicos o potencial de servicio inherente al activo. Por ello, la vida útil de un activo puede ser inferior a su vida económica. La estimación de la vida útil de un activo es una cuestión de criterio, basado en la experiencia que la entidad tenga con activos similares.

\section{- Método de Depreciación}

La Norma Internacional del Sector Público en mención establece que el método de depreciación utilizado reflejará el patrón con arreglo al cual se espera que sean consumidos, por parte de la entidad, los beneficios económicos futuros o el potencial de servicio del activo.

El método de depreciación aplicado a un activo se revisará, como mínimo, al término de cada periodo anual sobre el que se informe y, si hubiera habido un cambio significativo en el patrón esperado de consumo de los beneficios económicos futuros o potenciales de servicio incorporados al activo, se cambiará para reflejar el nuevo patrón. Dicho cambio se contabilizará como un cambio en una estimación contable, de acuerdo con la NICSP 3.

Pueden utilizarse diversos métodos de depreciación para distribuir el importe depreciable de un activo de forma sistemática a lo largo de su vida útil. Entre estos se encuentran:

- Método lineal: dará lugar a un cargo constante a lo largo de la vida útil del activo, siempre que su valor residual no cambie.

- Depreciación decreciente: en función del saldo del elemento dará lugar a un cargo que irá disminuyendo a lo largo de su vida útil.

- Por unidades de producción: dará lugar a un cargo basado en la utilización o producción esperada.

La entidad elegirá el método que más fielmente refleje el patrón esperado de consumo de los beneficios económicos o potencial de servicios futuros incorporados al activo. Dicho método se aplicará uniformemente de periodo a periodo, a menos que se haya producido un cambio en el patrón esperado de consumo de dichos beneficios económicos o potencial de servicios futuros. 
- Información a Revelar

En los estados financieros se deberá revelar para cada clase de elementos pertenecientes a la Propiedad, planta y equipo la siguiente información:

$\checkmark \quad$ Las bases de medición utilizadas para determinar el importe en libros bruto.

$\checkmark$ Los métodos de depreciación utilizados.

$\checkmark \quad$ Las vidas útiles 0 las tasas de depreciación utilizadas.

$\checkmark$ El importe bruto en libros y la depreciación acumulada (agregada con pérdidas acumuladas por deterioro del valor), al principio y final del periodo sobre el que se informa.

$\checkmark$ Una conciliación entre los importes en libros al principio y al final del periodo sobre el que se informa.

La entidad además presentará en sus estados financieros:

$\checkmark$ La existencia e importes en libros correspondientes a las restricciones de titularidad, así como los elementos de la propiedad, planta, y equipo que están afectas como garantías al cumplimiento de determinadas obligaciones (deudas).

$\checkmark$ El importe de los compromisos contractuales para la adquisición de elementos de la propiedad, planta, y equipo.

\section{ANÁLISIS DE RESULTADOS}

\section{ADAPTACIÓN A LA NORMATIVA}

En el trayecto de pasar de un Sistema de Contabilidad General de la Nación a NICSP, la entidad en estudio, Parque Metropolitano, tuvo obstáculos y desaciertos, pero pese a ello han logrado grandes metas enfrentando las dificultades presentadas, que han perfeccionado de manera increíble la calidad de la información financiera entregada por la entidad a la Contraloría, esperando entregar sus estados financieros próximos en la fecha correspondiente.

\section{- Resolución N¹6}

\section{Impacto}

$\checkmark$ El Parque Nacional enfrentó de buena manera la adaptación de las NICSP, teniendo un impacto a raíz de cómo la organización llevaba el control, en una proporción del $80 \%$ de los bienes de uso y bienes inmuebles, realizando lo que son sus actualizaciones (inventario) y depreciaciones en un programa. 


\section{- Proceso de Implementación}

\section{Debilidades}

$\checkmark$ Cuentan con bajos recursos económicos para poder incrementar lo que son los bienes inmuebles, y a su vez lo que conlleva este acontecimiento, como contratar a un especialista en tasación y realizar el estudio de mercado correspondiente para determinar su valor.

$\checkmark$ Están desfavorecidos por la normativa, ya que el rubro a la que se dedica la entidad no se encuentra mencionado ni detallado específicamente en las NICSP para ser contabilizado, lo que les dificultó clasificar estos activos.

$\checkmark \quad$ La activación y control de los bosques y áreas verdes se les hace difícil y complicada por los recursos inexistentes y porque no son propiedad del parque, pero sí los administran.

\section{Fortalezas}

$\checkmark$ Se destaca por haber llevado un registro anual de activos fijos antes de la implementación de la normativa. Como entidad los tenían bien definidos, tanto sus depreciaciones y las correcciones monetarias, logrando actualmente un ordenamiento más rápido y eficiente de estos activos como su tratamiento y valorización.

$\checkmark \quad$ Cuentan con el personal calificado y con experiencia en el tema de la nueva normativa que tienen diplomados en NICSP. Además de esto se realizaron capacitaciones programadas y organizadas por la Contraloría General de la República. También es importante destacar que cuentan con un área especializada en manejo y bienestar animal que se encarga del tratamiento y cuidado de los animales.

\section{- Estados Financieros}

\section{Objetivos Cumplidos}

$\checkmark \quad$ El Parque Nacional se destaca por ser uno de los primeros que presentaron los cinco estados financieros en el año 2015 como entidad piloto, cumpliendo con las fechas correspondientes y los requisitos que demanda la preparación de los EE.FF. Actualmente como entidad se preparan para presentar por segunda vez los EE.FF, fecha que fue aplazada por la Contraloría General de la República.

\section{- Plan de Convergencia}

\section{Tareas}

$\checkmark \quad$ Para la entidad siempre ha sido su tarea primordial cumplir con el objetivo principal del parque, siendo un propósito a conseguir muy importante para ellos. 
$\checkmark$ Además, otra tarea fundamental que tienen en espera es la implementación de la norma a todos los bienes pendientes que administran que aún no contabilizan, e ir actualizando los ya existentes tanto en infraestructura y cantidades que se encuentran en áreas verdes internas y externas del parque.

$\checkmark$ Actualmente aún están pendientes con las áreas verdes que corresponden a los bosques, por lo que tienen como tarea contabilizarlos porque hay bosques nuevos que se queman, que cortan, árboles que se caen, entre otras. Lo que pasa a ser es un tema complejo ya que los recursos son bajos para contratar a un tasador.

\section{Procedimientos}

$\checkmark$ Buscan regularizar lo que aún está pendiente, que sería el bienestar de trabajadores, un ámbito débil que presenta la entidad en este momento, buscando otorgarles más beneficios y seguridad a estos.

$\checkmark \quad$ Por otro lado, que es una gran preocupación para la entidad la falta de realizar un estudio de los parques urbanos que ocupa el Parque Nacional, si bien algunos se encuentran en concesión ya que son propiedad del SERVIU, son administrados por el parque, y por lo tanto se debe registrar como PPE, en la entidad en estudio, estando este tema aún pendiente ya que no ha tenido solución ni acuerdo.

\section{NORMATIVA EN PARTICULAR}

La entidad en estudio ha abordado la NICSP 17 sobre Propiedad, Planta y Equipo de manera cuidadosa con respecto a los animales de exhibición, pero presenta ciertas dificultades en algunos bienes de uso, pero que están en proceso de ser resueltos para ser contabilizados como tal.

\section{- Propiedad, Planta y Equipo}

\section{Principales Dificultades}

$\checkmark$ Una dificultad que se presenció en el Parque Nacional fue la recopilación de la información que llevaban registrada y controlada, y a su vez el análisis que se realizó para evaluar que correspondía a NICSP de Bienes de Uso y que no, para ser contabilizado bajo la normativa.

$\checkmark$ Respecto a lo que son los animales, el análisis fue mucho más complejo ya que la normativa no especifica este caso tan en particular, que son los animales de exhibición, lo que hizo que la interpretación de la norma fue mucho más compleja para el personal pertinente del área de contabilidad, teniendo que indagar 
más y evaluar bien este caso a través de un consenso para determinar lo criterios de aplicación por el cual la entidad registraría y contabilizaría estos bienes.

\section{Contabilización de Animales}

$\checkmark$ Se pudo aclarar que los animales que posee el Parque Nacional del Zoológico que se encuentran en exhibición para el público son contabilizados y tratados como Bienes de Uso, considerando un rango de valor mayor a 3 UTM para que sea activable.

\section{Elementos Bienes de Uso}

$\checkmark$ Todos los bienes son clasificados por su valor, vida útil y características propias, considerando que el valor debe ser mayor a 3 UTM para que sea activable, y la vida útil mayor a un año, y respecto a las características de un bien, que éste pueda ser controlado como su eje, válvulas muy caras, pero son enterradas para que no sean vistas más.

\section{Medición}

$\checkmark$ Se puede determinar que la valorización de las especies proviene directamente de los especialistas médicos, quienes asignan un valor según el mercado de la transacción de especies a nivel nacional e internacional, la longevidad y por su valor de importancia para el zoológico. Ahora bien, a través del tiempo se han recopilados estos valores creando una tabla de valores estimados, que es utilizada por la entidad.

$\checkmark \quad$ Las especies comienzan con un único valor inicial que posteriormente no cambia, y de ahí se va depreciando de acuerdo a los años de vida útil (longevidad). En el caso que se presente una muerte de algún animal, éste se da de baja.

$\checkmark$ El Departamento de Contabilidad se guía bajo una resolución de acta para contabilizar los valores de los animales que es realizada por los Doctores Veterinarios.

\section{Vida Útil}

$\checkmark$ Para este caso tan en particular se aclaró que si es posible determinar longevidad que corresponde a lo que se llama vida útil, que es determinada por los Doctores veterinarios del Zoológico utilizando sus propios criterios y juicio profesional. Respecto a las especies que se encuentran en cautiverio la longevidad es mayor a las especies de la vida silvestre, por los cuidados que reciben en el lugar de hábitat. 


\section{DISCUSIÓN DE RESULTADOS}

Las Normas de Información Contable para el Sector Público (NICSP), emitidas por la Federación Internacional de Contadores (IFAC), a través de la Resolución № 16, de fecha 16 de febrero del 2015, tuvo un gran impacto en Chile en las entidades públicas por la adopción y aplicación de estas normas, cuya finalidad era generar información financiera con el objetivo de suministrar información útil para la toma de decisiones y constituir un medio para la rendición de cuentas de los recursos que han sido asignados a cada entidad. Esta nueva normativa implementada por el sector público tiene similitud con la que aplica el sector privado, utilizando grandes estándares internacionales que permiten la comparación de la información con otros países que han adoptado la NICSP.

En la situación que se encontraba la institución en estudio, Parque Metropolitano de Santiago, en especial el Zoológico Nacional, la implementación de este plan de convergencia referido a las Normas Internacionales Contable para el Sector Público, presentó un gran cambio en su sistema contable que fue establecido por la nación. Sin embargo, no tuvo un gran impacto, ya que tenían un registro individual de los bienes de uso de la entidad, más sus depreciaciones, y por último las correcciones monetarias que hoy en día no se aplica, debiendo adecuar y reorientar esta información a la normativa y formatos de estados financieros presentados por la Contraloría General de la República.

Es relevante que para cumplir con los procedimientos y tareas que se deben llevar a cabo al aplicar estas normas internaciones se cuente con el personal técnico que tenga conocimiento del tema, contando así con profesionales especializados y capacitados en el tema de NICSP tanto en su área contable, inventario, y como anexo en el área de biología, también contando con los doctores veterinarios expertos en el tratamiento biólogo de los animales. Estos últimos, tienen un rol fundamental en la generación de la información que se registra contablemente, ya que informan al departamento de inventario cuál es la cantidad de cada animal que se tiene de las diferentes especies que posee el zoológico, dos veces al año la información del inventario es actualizada, facilitándole así al área de contabilidad el precio de los animales recién nacidos, los de canje, y los donados siempre especificando cuántos animales son los que ingresan y egresan, (además de su longevidad que en términos contables se reconoce como vida útil, este dato de cada especie ya es determinado por libros biológicos).

La institución en estudio al tener como función ser una "Entidad Piloto" elegida por la Contraloría General de la República, para iniciar este proceso de convergencia en el año 2011, a través del plan de implementación con sus distintas etapas, se fueron identificando los factores críticos, los riesgos, y las estrategias de mitigación, teniendo esto como finalidad incorporar la exigencia de la preparación de los Estados Financieros. Como entidad piloto fueron adecuando y modificando la información contable respecto a su situación en particular, siendo uno de los primeros en entregar y presentar sus cincos estados financieros, respetando la fecha y la formalidad correspondiente de cada uno, en el año 2015, sin resultar con contingencia entre un valor y otro contabilizado. 
Para el plan futuro de esta entidad, faltan desarrollar procedimientos y tareas que están pendientes aún en el proceso, como lo es el bienestar de los trabajadores. Por otro lado, aún deben regularizar los bienes que les faltan, como los parques urbanos administrados por la entidad pero que no pertenecen a su patrimonio. Respecto a las áreas verdes, que vienen siendo los bosques, es un escenario complicado, ya que estos aún están en proceso de ser contabilizados, presentándose en ellos distintos casos, como bosques nuevos, bosques que se queman, que se cortan, árboles que se caen, entre otros. Paralelamente se les presenta la debilidad más destacada que afecta a estos procedimientos y tareas, que es la poca capacidad de recursos que cuenta la entidad para poder resolverlos.

Finalmente los animales, seres vivos exhibidos para el público tal como se da en el zoológico, es un caso súper particular e interesante que la NICSP 17 no detalla específicamente, por lo que pasa a ser este acontecimiento una tarea complicada para la entidad en estudio en la comprensión de la normativa. Pero respecto a los análisis estos animales, estos se contabilizan como bienes de uso (PPE), contando solo con un valor único que se obtiene a través de un estudio de mercado, ya que la entidad no compra ni vende los animales (lo anterior viene siendo la medición inicial aún solo valor pero no tienen un valor posterior).

\section{CONCLUSIONES}

La Contraloría General de la República, mediante el oficio № 54977 , del 15 de septiembre de 2010, incorpora a la Normativa del Sistema de Contabilidad General de la Nación el Capítulo Quinto "Estados Financieros", cuyo objetivo es suministrar a las entidades del sector público información útil para la toma de decisiones y contribuir a la transparencia y rendición de cuentas de los recursos asignados, incrementando la calidad y el detalle de la información financiera. En esta primera etapa se conformó un grupo de servicios públicos, los cuales fueron designados como "Entidad Piloto", quienes tendrían como función ser contraparte de la Contraloría en el análisis y evaluación del impacto del cambio normativo, el cual comenzó a aplicarse para ellas y el resto de los servicios a contar del año 2015, presentando sus primeros estados financieros bajo NICSP.

Una de estas entidades piloto es el Parque Metropolitano, la cual ha sido capaz de llevar a cabo las etapas del plan de convergencia mediante la capacitación del personal y las consultas a la CGR sobre los procedimientos y tareas nuevas que van surgiendo mediante este plan de convergencia. También, cumplió con este proceso de implementación entregando los Estados Financieros en la fecha correspondiente indicada y bajo los formatos establecidos por la CGR.

Respecto a los objetivos específicos de la presente investigación, sobre las normativas asociadas a los bienes considerados como activos materiales en la Norma Internacional de Contabilidad para Sector Público, se identificó que la normativa que aplica en este caso tan en particular, que son los animales de exhibición del zoológico, es la NICSP 17 sobre Propiedad, Planta y Equipo. 
En relación con lo anterior sobre el objetivo general de la investigación se puede destacar que los animales de exhibición son reconocidos como activos, ya que la entidad obtiene beneficios futuros económicos, ya que son significativos para la situación financiera.

Sobre el objetivo general de la investigación se puede destacar que los animales de exhibición son reconocidos como activos, ya que la entidad obtiene beneficios futuros económicos, ya que son significativos para la situación financiera.

El tratamiento de los animales que son donados a la entidad por otras similares se adquieren por un costo nulo 0 insignificante, implicando que el costo de la partida (o rubro) es su valor razonable en la fecha de su adquisición, por lo que no constituye una revaluación.

En el caso de canje de los animales, es conocido como intercambio de activos que se cambian por otro activo similar cualquiera, con un uso parecido dentro de la misma línea de actividad y con un valor similar al entregado. El costo de adquisición de tal elemento se medirá por el valor razonable del activo recibido, que es equivalente al valor razonable del activo entregado, ajustado por el importe de cualquier eventual cantidad de efectivo u otros medios líquidos entregados adicionalmente.

Finalmente, en el caso de los animales que son producidos por la misma entidad, el costo de éstos será determinado utilizando los mismos principios que los animales que son adquiridos del exterior, o sea por canje o donación, entonces su costo será a valor razonable. Respecto a la longevidad que viene siendo la vida útil de estos animales en cautiverio son determinados por libros biológicos.

\section{BIBLIOGRAFÍA}

- Contraloría General de la República. (2012). Obtenido de NICSP: http:// www.contraloria.cl/NewPortal2/portal2/appmanager/ExtranetCGR/NICSP

- Curiqueo A, M., \& Estay M, L. (2016). Análisis de la Implementación de la Resolución 16/2015, de la Contraloría General de la República referido a los bienes de uso en un servicio público de la región de Valparaíso, periodo 2016. Valparaíso, Chile.

- Federación Internacional de Contadores. (2008). Obtenido de Información sobre Antecedentes de la IFAC y otros Recursos: http://www.ifac. org/system/files/publications/files/ES_A4_IFAC_Background_Info.pdf

- Ley Nro. 18.575. (2008). Ley Orgánica Constitucional de Beses Generales de la Administración del Estado. Santiago de Chile. 
- Mellado, L. T. (2013). Normas Internacionales de Contabilidad del Sector Público: Convergencia e Impacto en Chile. Punta Arenas, Chile: La Prensa Austral IMPRESOS.

- R., D. F. (2003). Conceptos de la Administración Estratégica. México: Pearson Education.

- Superintendencia de Valores y Seguros . (05 de 2011). Obtenido de Proceso de convergencia a Estándares Internacionales de Contabilidad : $\quad$ https://www.svs.cl/portal/prensa/604/articles-13385_doc_pdf.pdf

- Vilches B, M. (2015). Análisis del Proceso de Convergencia de las Normas Internacionales de Contabilidad para el Sector Público, Enfocado en el Capítulo IV de la Resolución CGR Nro. 16, en un servicio público piloto para el periodo 2013. Valparaíso, Chile 